\title{
Research Note \\ The identification of GLMP 160 as a new post-AGB candidate with [WC]-spectrum ${ }^{\star}$
}

\author{
C. B. Pereira and M. Machado
}

Observatório Nacional-MCT, Rua José Cristino, 77, CEP 20921-400, São Cristóvão, Rio de Janeiro-RJ, Brazil

Received 7 April 2003 / Accepted 19 May 2003

\begin{abstract}
We report spectroscopic observations of a new post-AGB star with WR spectrum not identified yet. This star was found during a southern spectroscopy survey of post-AGB candidates selected from Garcia-Lario et al. (1990). This star, GLMP 160, shows the main characteristics of a [WC] spectrum with strong C III 4647, C IV 5808 and He II 4686. In addition the spectrum presents a strong [O III]5007 $\AA$ line. From the line ratio using the carbon lines and the measurements of $F W H M$ we proposed that GLMP 160 has spectral characteristics of a [WC6-7] subtype. Here we present some of the main spectroscopic features and discuss the evolutionary status. We also present line intensities and equivalent widths of the identified lines.
\end{abstract}

Key words. stars: emission line, [WC]-stars

\section{Introduction}

We present spectroscopic observations of a peculiar emissionline star, GLMP 160, which was first recognized as a planetary nebulae candidate after the near infrared photometric survey of García-Lario et al. (1997). GLMP 160 is also known as PM 1-23 (Preite-Martinez 1988) who was the first to associate it as a planetary nebulae candidate with a IRAS source, IRAS 06518-1041. Later on, García-Lario et al. (1990) presented infrared colors of several IRAS sources in order to separate objects between AGB and post-AGB stars to protoplanetary nebulae and planetary nebulae. GLMP 160 was selected to observe in the low resolution mode, among other stars, from a larger sample of García-Lario et al. (1997). We selected some stars from this sample in order to make a more accurate classification of their evolutionary status using spectroscopic data of some objects in the transition phase (post-AGB stars) and the planetary nebulae candidates.

The spectroscopic analysis of GLMP 160 indicate a hydrogen-deficient central star with spectral characteristics of a carbon Wolf-Rayet ([WC] central star) with an associated nebulae. In this paper we present a discussion of the analysis of the spectroscopic observations and we comment on the criteria used to classify GLMP 160 as a post-AGB star with a [WC6-7] central star.

Send offprint requests to: C. B. Pereira, e-mail: claudio@obsn.on.br

* Based on observations made with the $1.52 \mathrm{~m}$ telescope at the European Southern Observatory (La Silla, Chile) under the agreement with the Observatório Nacional, Brazil.

\section{Observations and reduction}

Spectroscopic observations were performed using a Boller \& Chivens spectrograph at the Cassegrain focus of the ESO $1.52 \mathrm{~m}$ telescope in La Silla (Chile) at 25 December, 2002. A UV-flooded thinned Loral Lesser CCD \#39 $(2048 \times 2048$, $15 \mu \mathrm{m} /$ pixel) was used as the detector. The setup employed is the same as that used in the discovery a new Wolf-Rayet (SPH 2) some years ago (Pereira et al. 1998). We used the grating \#23 with $600 \mathrm{l} / \mathrm{mm}$ providing a resolution of $4.6 \AA$ in the range $\lambda \lambda 3500 \AA-8000 \AA$. The spectra were reduced using standard IRAF tasks, from bias subtraction and flat-field correction, through spectral extraction and wavelength and flux calibration. Spectrophotometric standards from Oke (1974) and Hamuy et al. (1994) were also observed.

The flux of emission lines have been measured by the conventional method adjusting a Gaussian function to the line profile thereby obtaining the intensity, the central wavelength and the line width at half power level. Uncertainties in the line intensities come mainly from the position of the underlying continuum. We estimate the flux error to be about $20 \%$ for the weak lines (line fluxes $\approx 10$ on the scale of $\mathrm{H} \beta=100$ ) and about $10 \%$ for stronger lines. Figure 1 shows the reduced spectra.

A finding chart of the object is present in Fig. 2. The equatorial coordinates of GLMP 160 are $\alpha(2000.0)=06^{\mathrm{h}} 54^{\mathrm{m}} 37^{\mathrm{s}}$ and $\delta(2000.0)=-10^{\circ} 45^{\prime} 37^{\prime \prime}$. The $V$ magnitude was derived convolving the calibrated spectrum with a $V$ filter profile, giving 15.2. 


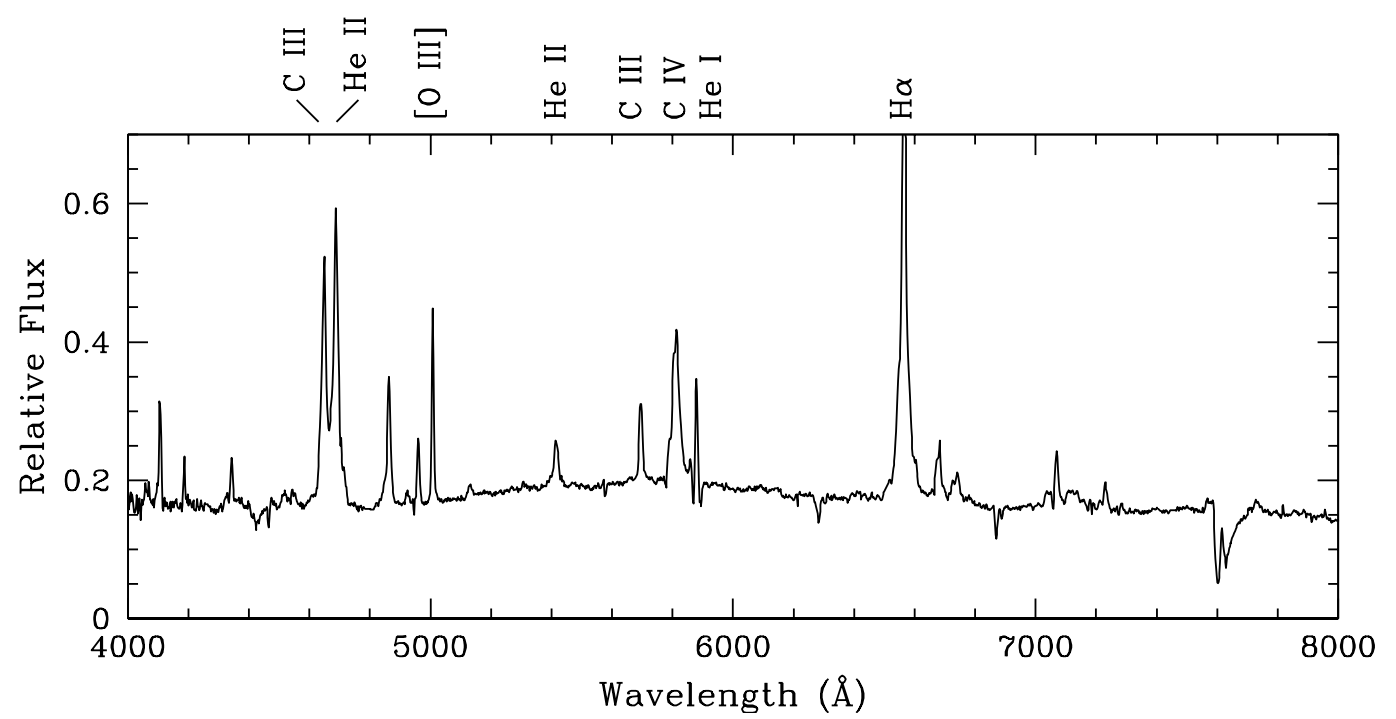

Fig. 1. Optical spectrum of GLMP 160. Notice the presence of C III4647 A and C IV5812 A.

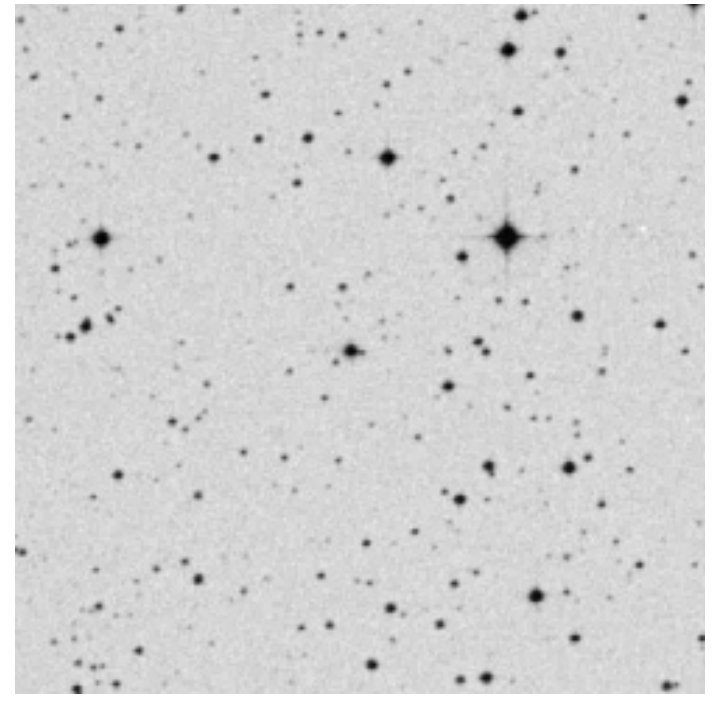

Fig. 2. Finding chart of the region around GLMP 160. GLMP 160 is at the center of the field. The field has dimensions of $7^{\prime} \times 7^{\prime}$. North is at the top and east is to the left.

\section{The spectrum of GLMP 160}

In Table 1 we list the lines that we have identified as well as their values of line fluxes, equivalent widths and FWHM. The spectrum called our atention due the presence of the lines C III $\lambda 5696 \AA$ and the doublet C IV $\lambda \lambda 5802,12 \AA$. These lines are typical of carbon Wolf-Rayet stars and are used as the main criterium to classify these stars. We also identify several other lines of C III, another line of C IV and two lines of He II. All these lines are wide and should have formed in the wind of central star. The nebular component can be identified due to the presence of the forbbiben lines of [O III] $\lambda \lambda 4959,5007$ and H I Balmer lines with narrower profiles. The helium line at $\lambda 5876 \AA$ should also be nebular because its $F W H M$ value is comparable to other nebular lines.
Table 1. Observed emission line flux $F(\lambda)$ relative to $\mathrm{H} \beta=100$, equivalent widths $\left(W_{\lambda}\right)$ and full-width half-maximum $(F W H M)(\AA)$ of GMLP 160.

\begin{tabular}{|c|c|c|c|c|}
\hline Wavelength & Identification & $F(\lambda)$ & $W_{\lambda}(\AA)$ & $F W H M(\AA)$ \\
\hline 4056 & C III & 4.8 & 0.7 & 5.4 \\
\hline 4101 & $\mathrm{H} \delta$ & 68.1: & 11.1 & 9.5 \\
\hline 4186 & C III & 13.3 & 2.0 & 5.0 \\
\hline 4326 & C III & 9.0 & 1.4 & 12.6 \\
\hline 4340 & $\mathrm{H} \gamma$ & 21.8 & 3.3 & 8.1 \\
\hline 4514 & C III & 15.8 & 2.5 & 21.1 \\
\hline 4543 & CIV & 11.9 & 2.0 & 16.9 \\
\hline 4647 & C III & 285.2 & 42.9 & 24.7 \\
\hline 4686 & He II & 323.7 & 49.5 & 24.1 \\
\hline 4861 & $\mathrm{H} \beta$ & 100.0 & 15.4 & 14.1 \\
\hline 4921 & $\mathrm{He} \mathrm{I}$ & 9.2 & 1.4 & 11.0 \\
\hline 4959 & [O III] & 39.7 & 4.7 & 8.2 \\
\hline 5007 & [O III] & 108.3 & 16.8 & 8.2 \\
\hline 5131 & C III & 1.2 & 1.4 & 15.5 \\
\hline 5411 & He II & 56.0 & 7.5 & 21.5 \\
\hline 5696 & C III & 61.7 & 7.7 & 15.1 \\
\hline 5812 & CIV & 286.4 & 38.4 & 31.2 \\
\hline 5876 & $\mathrm{He} \mathrm{I}$ & 84.6 & 13.4 & 9.6 \\
\hline 6563 & $\mathrm{H} \alpha$ & 564.3 & 79.2 & 16.5 \\
\hline 6678 & $\mathrm{He}$ I & 19.7 & 2.8 & 11.1 \\
\hline 6683 & He II & 31.2 & 4.4 & 12.9 \\
\hline 6725 & $\mathrm{C}_{\text {III }}$ & 19.6 & 2.9 & 18.2 \\
\hline 6740 & C III & 29.0 & 4.2 & 16.1 \\
\hline 7041 & C III & 27.4 & 4.3 & 24.5 \\
\hline 7065 & He I & 44.4 & 7.0 & 12.3 \\
\hline 7119 & C II? & 24.5 & 4.4 & 42.3 \\
\hline 7213 & C III & 14.7 & 2.4 & 13.3 \\
\hline 7230 & C II? & 25.3 & 4.0 & 18.9 \\
\hline
\end{tabular}

\section{The evolutionary status of GLMP 160}

The origin of hydrogen-deficient post-AGB stars is still not well understood (Koesterke 2001). In this group a large number of objects with central stars with spectral characteristics of carbon Wolf-Rayet stars ([WC] central stars) 


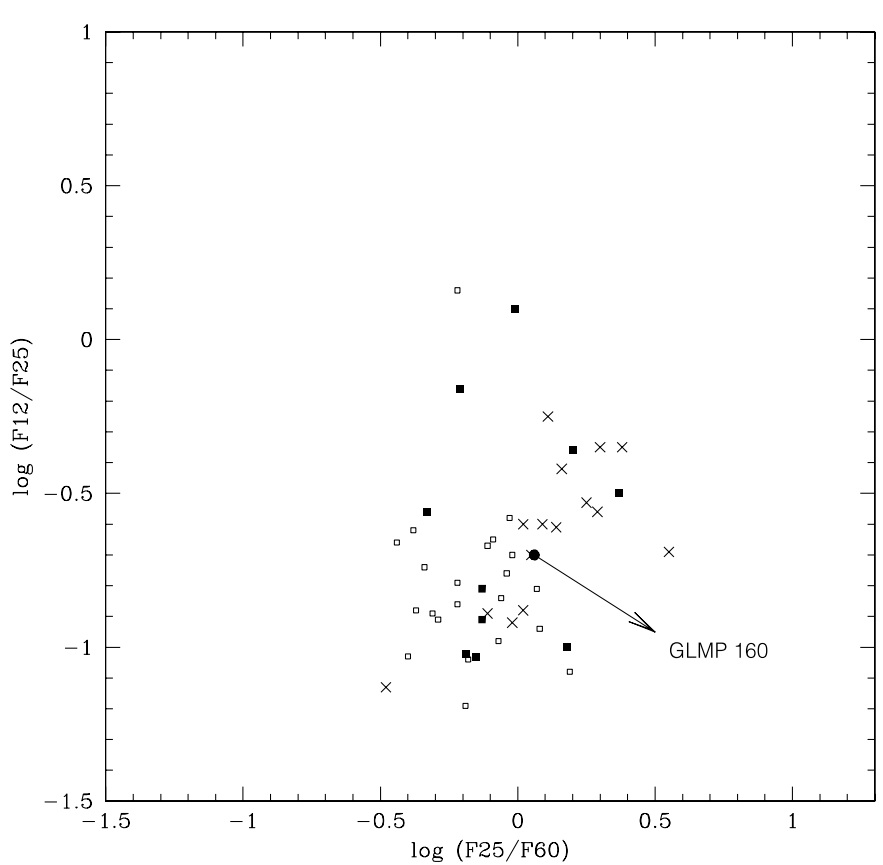

Fig. 3. Position of [WC] subclasses and GLMP 160 in the IRAS twocolour diagram. Position of [WC 2-4] is shown by the open square points; [WC 5-7] filled square points; [WC 8-11] diagonal cross points. The position GLMP 160 is given by filled circle.

have been recognized. They are found at positions in the Hertzprung-Russel diagram which corresponds to evolutionary sequences of low and intermediate mass stars (Herwig 2001). The [WC] central stars are distributed in spectral subtypes such as the Population I WC stars. However, contrary to Population I, the [WC] central stars are concentrate in the extreme subtypes [WC2-4] and [WC9-11] with a apparent lack of [WC5-7] stars. Our spectroscopic data indicate that the central star of GLMP 160 shows characteristics of intermediate subtypes. The classification criterium proposed by Crowther et al. (1998) uses as a primary classification diagnostic the CIV $\lambda \lambda 5801,12 / \mathrm{C}$ III $\lambda 5696$ ratio. This ratio for GLMP 160 is 0.69 and correponds to a [WC6] subtype. The value of the FWHM of the blend CIV $\lambda 5808$ is also an indication of this subtype. Other interesting point is the probable presence of two lines at $7120 \AA$ and at $7231 \AA$ having flat and broad shape which are typical of wind of
Wolf-Rayet stars. In these wavelengths there are two lines of C II (7119 $\AA$ and $7231 \AA$ - multiplets 20 and 3 respectivelly) that would indicate a [WC7] subtype. To confirm the presence of C II would be important to identify another line of C II such as $4267 \AA$ which is used as a classification criterium. For this we would need observations with higher resolution, due to the high winds velocities the identification of weaker lines becomes difficult.

Figure 3 shows the IRAS colour-colour diagram for all stars of the sample of Tylenda et al. 1993. According to Acker et al. (1996) this diagram shows a trend of the colours becoming redder from the later to the earlier subtypes and can be compared with the post-AGB evolution. The [WC9-11] objects evolve to [WC2-4] subtypes with a fast intermediate phase [WC5-7]. The place of GLMP 160 in this diagram corresponds to the intermediate subtype.

\section{Summary and conclusions}

Based on a spectroscopic survey between $4000 \AA-8000 \AA$ of post-AGB stars candidates selected from García-Lario et al. (1997) we discovered a emission-line star which presents spectral characteristics of a Wolf-Rayet star. Based on the line ratio CIV $\lambda \lambda 5801,12 / \mathrm{C}$ III $\lambda 5696$, the measurements of the FWHM and IRAS colors, we proposed that GLMP 160 has spectral characteristics of a intermediate [WC6-7] subtype.

\section{References}

Acker, A., Górny, S. K., \& Cuisinier, F. 1996, A\&A, 305, 944

Crowther, P. A., de Marco, O., \& Barlow, M. J. 1998, MNRAS, 296, 367

García-Lario, P., Manchado, P., Suso, S. R., Pottasch, S. R., \& Olling, R. 1990, A\&AS, 82, 497

García-Lario, P., Manchado, P., Pych, W., et al. 1997, A\&AS, 126, 479

Hamuy, M., Suntzeff, N. B., Heathcote, S. R., et al. 1994, PASP, 106, 566

Herwig, F. 2001, Ap\&SS 2001, 275, 15

Koesterke, L. 2001, Ap\&SS, 275, 41, 2001

Oke, J. B. 1974, ApJS, 27, 21

Pereira, C. B., Machado, M. A. D., Landaberry, S. J. C., \& Conceição, F. 1998, A\&A, 338, L91, 1998

Preite-Martinez, A. 1988, A\&AS, 76, 317

Tylenda, R., Acker, A., \& Stenholm, B. 1993, A\&AS, 102, 595. 